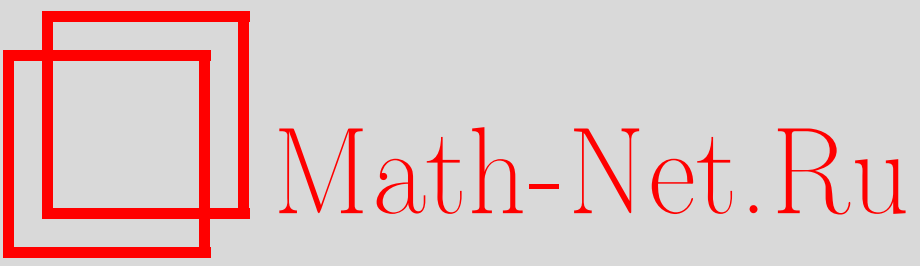

Л. Г. Рыбников, Централизаторы некоторых квадратичных элементов в алгебрах Пуассона-Ли и метод сдвига инвариантов, УМH, 2005, том 60, выпуск 2, $173-$ 174

DOI: https://doi.org/10.4213/rm1421

Использование Общероссийского математического портала Math-Net.Ru подразумевает, что вы прочитали и согласны с пользовательским соглашением http://www . mathnet.ru/rus/agreement

Параметры загрузки:

IP : 35.174 .16 .151

26 апреля 2023 г., 02:19:58 


\title{
ЦЕНТРАЛИЗАТОРЫ НЕКОТОРЫХ КВАДРАТИЧНЫХ ЭЛЕМЕНТОВ В АЛГЕБРАХ ПУАССОНА-ЛИ И МЕТОД СДВИГА ИНВАРИАНТОВ
}

\author{
Л. Г. РЫБников
}

1. Пусть $\mathfrak{g}$ - полупростая комплексная алгебра Ли и $\mathfrak{h} \subset \mathfrak{g}$-ее картановская подалгебра. Универсальная обертьвающая алгебра $U(\mathfrak{g})$ имеет возрастающую фильтрацию по степени выражения через образующие. По теореме Пуанкаре-Биркгофа-Витта ассоциированная градуированная алгебра есть симметрическая алгебра $S(\mathfrak{g})=\mathbb{C}\left[\mathfrak{g}^{*}\right]$. Коммутатор в $U(\mathfrak{g})$ задает скобку Пуассона-Ли $\{\cdot, \cdot\}$ в $S(\mathfrak{g})$. На образующих $x, y \in \mathfrak{g}$ имеем $\{x, y\}=[x, y]$.

Метод сдвига инвариантов позволяет строить коммутативные (относительно $\{\cdot, \cdot\})$ подалгебры в $S(\mathfrak{g})$. Этот метод состоит в следующем. Для любого регулярного элемента $h_{0} \in \mathfrak{h} \subset$ $\mathfrak{g}=\mathfrak{g}^{*}$ подалгебра $A\left(h_{0}\right) \subset S(\mathfrak{g})$, порожденная $\mathfrak{g}$-инвариантами в $S(\mathfrak{g})=\mathbb{C}\left[\mathfrak{g}^{*}\right]$ и их производными всех порядков вдоль $h_{0}$, коммутативна и имеет максимально возможную степень трансцендентности, равную $\frac{1}{2}(\operatorname{dim} \mathfrak{g}+\mathrm{rk} \mathfrak{g})\left(\right.$ см. [1]). Более того, подалгебры $A\left(h_{0}\right)$ являются максимальными коммутативными подалгебрами в $S(\mathfrak{g})$ относительно скобки Пуассона-Ли [2]. Подалгебры $A\left(h_{0}\right)$ названы в [3] подалгебрами Мищенко-Фоменко.

Пусть $\Delta \subset \mathfrak{h}^{*}$ - система корней алгебры $\mathfrak{g}, \Delta_{+}-$множество положителшных корней, $\alpha_{1}, \ldots, \alpha_{l}$ - простые корни. Зафиксируем инвариантное невырожденное скалярное умножение $(\cdot, \cdot)$ на $\mathfrak{g}$ и выберем в каждом корневом пространстве $\mathfrak{g}_{\alpha}, \alpha \in \Delta$, элемент $e_{\alpha}$ так, чтобы $\left(e_{\alpha}, e_{-\alpha}\right)=1$. Положим $h_{\alpha}:=\left[e_{\alpha}, e_{-\alpha}\right]$, тогда для любого $h \in \mathfrak{h}$ имеем $\left(h_{\alpha}, h\right)=\alpha(h)$. Элементы $e_{\alpha}(\alpha \in \Delta)$ вместе с элементами $h_{\alpha_{1}}, \ldots, h_{\alpha_{l}} \in \mathfrak{h}$ образуют базис пространства $\mathfrak{g}$.

Линейная и квадратичная части подалгебр Мищенко-Фоменко допускают следующее описание [4]: $A\left(h_{0}\right) \cap \mathfrak{g}=\mathfrak{h}, A\left(h_{0}\right) \cap S^{2}(\mathfrak{g})=S^{2}(\mathfrak{h}) \oplus Q\left(h_{0}\right)$, где $Q\left(h_{0}\right)=\left\{\sum_{\alpha \in \Delta_{+}} \frac{\alpha(h)}{\alpha\left(h_{0}\right)} e_{\alpha} e_{-\alpha} \mid\right.$ $h \in \mathfrak{h}\}$.

Основным результатом данной работы является следующая

ТЕорема 1. При общих значениях параметра $h_{0} \in \mathfrak{h}$ (т.е. для $h_{0}$, лежащих в дополнении счетного числа собственных замкнутых по Зарисскому подмножеств в $\mathfrak{h}$ ) алгебра $A\left(h_{0}\right)$ есть централизатор пространства $Q\left(h_{0}\right)$ в $S(\mathfrak{g})$ относительно скобки Пуассона-Ли.

В [5], [6] подалгебры Мищенко-Фоменко были подняты в универсальную обертьвающую алгебру, т.е. построены такие коммутативные подалгебры $\mathscr{A}\left(h_{0}\right) \subset U(\mathfrak{g})$, что $\operatorname{gr} \mathscr{A}\left(h_{0}\right)=A\left(h_{0}\right)$. Мы выведем из теоремы 1 следующее утверждение.

Теорема 2. При общих значениях параметра $h_{0} \in \mathfrak{h}$ существует не более одной коммутативной подалгебры $\mathscr{A}\left(h_{0}\right) \subset U(\mathfrak{g})$, для которой gr $\mathscr{A}\left(h_{0}\right)=A\left(h_{0}\right)$.

В случае $\mathfrak{g}=\mathfrak{g l}_{n}$ это было доказано А.А. Тарасовым [7] для любого регулярного $h_{0} \in \mathfrak{h}$.

2. Докажем теорему 1 . Заметим, что для любого $n$ множество $E_{n} \subset \mathfrak{h}$ таких $h_{0} \in \mathfrak{h}$, что централизатор подпространства $Q\left(h_{0}\right)$ в $S^{n}(\mathfrak{g})$ относительно скобки Пуассона-Ли имеет размерность, большую $\operatorname{dim} A\left(h_{0}\right) \cap S^{n}(\mathfrak{g})$ (т.е. строго содержит пространство $A\left(h_{0}\right) \cap S^{n}(\mathfrak{g})$ ), замкнуто по Зарисскому в $\mathfrak{h}$. Поэтому достаточно доказать, что $E_{\boldsymbol{n}} \neq \mathfrak{h}$ для любого $n$. Иначе говоря, достаточно доказать существ ование $h_{0} \in \mathfrak{h}$, удовлетворяющего условию теоремы.

Лемма 1. Существуют такие $h_{0}, h \in \mathfrak{h}$, что числа $\frac{\alpha(h)}{\alpha\left(h_{0}\right)}\left(\alpha \in \Delta_{+}\right)$линейно независимы над полем $\mathbb{Q}$.

ДокАЗАтЕльство. Выберем $h_{0}$ так, чтобы для простых корней $\alpha_{i}$ значения $\alpha_{i}\left(h_{0}\right)$ были алгебраически независимы над полем $\mathbb{Q}$. Так как никакие два положительных корня не пропорциональны, то числа $\frac{1}{\alpha\left(h_{0}\right)}, \alpha \in \Delta_{+}$, линейно независимы над $\mathbb{Q}$. Выберем $h$ так, чтобы значения $\alpha(h)$ были ненулевыми рациональными числами. Тогда числа $\frac{\alpha(h)}{\alpha\left(h_{0}\right)}, \alpha \in \Delta_{+}$, линейно независимы над $\mathbb{Q}$.

Работа выполнена при поддержке фонда CRDF (грант RM1-2543). 
Выберем элемент $\gamma \in \mathfrak{g}^{*}$ такой, что $\gamma\left(h_{\alpha_{i}}\right)=1$ для простых корней $\alpha_{i}$ и $\gamma\left(e_{\alpha}\right)=0$ для $\alpha \in \Delta$. Зададим скобку Пуассона $\{\cdot, \cdot\}_{\gamma}$ на $S(\mathfrak{g})$ следующим образом: для образующих $x, y \in \mathfrak{g}$ положим $\{x, y\}_{\gamma}=\gamma([x, y])$. При любом $t \in \mathbb{C}$ линейная комбинация $t\{\cdot, \cdot\}+(1-t)\{\cdot, \cdot\}_{\gamma}$ является скобкой Пуассона на $S(\mathfrak{g})$, причем для $t \neq 0$ соответствующие алгебры Пуассона изоморфны. А именно, пусть $S(\mathfrak{g})_{t}$ - алгебра $S(\mathfrak{g})$ со скобкой Пуассона $t\{\cdot, \cdot\}+(1-t)\{\cdot, \cdot\}_{\gamma}$; тогда при $t \neq 0$ изоморфизм $\psi_{t}: S(\mathfrak{g})_{1} \rightarrow S(\mathfrak{g})_{t}$ задается на образующих $x \in \mathfrak{g}$ следующим образом: $\psi_{t}(x)=t^{-1} x+t^{-2}(1-t) \gamma(x)$. Очевидно, что $\psi_{t}\left(Q\left(h_{0}\right)\right)=Q\left(h_{0}\right)$.

Лемма 2. Для некоторого $h_{0} \in \mathfrak{h}$ централизатор пространства $Q\left(h_{0}\right)$ в $S(\mathfrak{g})_{0}$ имеет степень трансцендентности не больше $\frac{1}{2}(\operatorname{dim} \mathfrak{g}+\mathrm{rk} \mathfrak{g})$.

ДокАЗАТЕльство. Выберем $h_{0}$ и $h$ как в лемме 1 и положим $q=\sum_{\alpha \in \Delta_{+}} \frac{\alpha(h)}{\alpha\left(h_{0}\right)} e_{\alpha} e_{-\alpha} \in$ $Q\left(h_{0}\right)$. Для любого $f \in S(\mathfrak{g})$ имеем $\{q, f\}_{\gamma}=\sum_{\alpha \in \Delta_{+}} \gamma\left(h_{\alpha}\right) \frac{\alpha(h)}{\alpha\left(h_{0}\right)}\left(e_{-\alpha} \frac{\partial f}{\partial e_{-\alpha}}-e_{\alpha} \frac{\partial f}{\partial e_{\alpha}}\right)$. В частности,

$$
\left\{q, \prod_{i=1}^{l} h_{\alpha_{i}}^{m_{i}} \prod_{\alpha \in \Delta_{+}} e_{\alpha}^{n_{\alpha}} e_{-\alpha}^{n_{-\alpha}}\right\}_{\gamma}=\sum_{\alpha \in \Delta_{+}} \gamma\left(h_{\alpha}\right) \frac{\alpha(h)}{\alpha\left(h_{0}\right)}\left(n_{-\alpha}-n_{\alpha}\right) \prod_{i=1}^{l} h_{\alpha_{i}}^{m_{i}} \prod_{\alpha \in \Delta_{+}} e_{\alpha}^{n_{\alpha}} e_{-\alpha}^{n_{-\alpha}} .
$$

Для каждого $\alpha=\sum_{i=1}^{l} m_{i} \alpha_{i} \in \Delta_{+}$имеем $\gamma\left(h_{\alpha}\right)=\sum_{i=1}^{l} m_{i} \in \mathbb{Q} \backslash\{0\}$. Так как числа $\frac{\alpha(h)}{\alpha\left(h_{0}\right)}$ линейно независимы над $\mathbb{Q}$, то правая часть равенства $(1)$ равна нулю тогда и только тогда, когда для любого $\alpha \in \Delta_{+}$вьполнено $n_{\alpha}-n_{-\alpha}=0$. Это значит, что централизатор элемента $q$ в $S(\mathfrak{g})_{0}$ состоит из линейных комбинаций мономов, имеющих равную степень по $e_{\alpha}$ и $e_{-} \alpha$ для любого $\alpha \in \Delta_{+}$, т.е. порожден элементами $h_{\alpha_{i}}$ и $e_{\alpha} e_{-\alpha}\left(\alpha \in \Delta_{+}\right)$, и, следовательно, имеет степень трасцендентности $\frac{1}{2}(\operatorname{dim} \mathfrak{g}+\mathrm{rk} \mathfrak{g})$.

Из леммы 2 следует, что централизатор пространства $Q\left(h_{0}\right)$ в $S(\mathfrak{g})_{t}$ имеет степень трансцендентности не больше $\frac{1}{2}(\operatorname{dim} \mathfrak{g}+\operatorname{rk} \mathfrak{g})$ при общем $t$, а значит, и при любом $t$, так как при $t \neq 0$ пуассоновы алгебры $S(\mathfrak{g})_{t}$ изоморфны. Пусть $Z \subset S(\mathfrak{g})$ - централизатор пространства $Q\left(h_{0}\right)$ в $S(\mathfrak{g})_{1}$. Поскольку $\operatorname{tr} \operatorname{deg}(Z) \leqslant \operatorname{tr} \operatorname{deg}\left(A\left(h_{0}\right)\right)$ и $A\left(h_{0}\right) \subset Z$, любой элемент алгебры $Z$ алгебраичен над $A\left(h_{0}\right)$. Следовательно, так как алгебра $A\left(h_{0}\right)$ коммутативна относительно скобки Пуассона-Ли, алгебра $Z$ также коммутативна (см., например, [8; лемма 1$])$. Но $A\left(h_{0}\right)$ - максимальная коммутативная подалгебра в $S(\mathfrak{g})$, поэтому $Z=A\left(h_{0}\right)$. Теорема 1 доказана.

3. Докажем теорему 2. В [3] доказано, что пространство $A\left(h_{0}\right)^{(2)}=\mathbb{C}+\mathfrak{h}+S^{2}(\mathfrak{h})+Q\left(h_{0}\right)$ может быть поднято до коммутативного подпространства $\mathscr{A}\left(h_{0}\right)^{(2)} \subset U(\mathfrak{g})^{(2)}$ единственным способом (при помощи отображения симметризации). Из теоремы 1 следует, что при общем $h_{0}$ любое поднятие $\mathscr{A}\left(h_{0}\right) \subset U(\mathfrak{g})$ алгебры $A\left(h_{0}\right)$ есть централизатор пространства $\mathscr{A}\left(h_{0}\right)^{(2)}$ в алгебре $U(\mathfrak{g})$. Теорема 2 доказана.

Я благодарю Э. Б. Винберга за внимание к работе и полезные обсуждения.

\section{СПИСОК ЛИТЕРАТУРЫ}

[1] А. С. Мищенко, А. Т. Фоменко // Труды семинара по векторному и тензорному анализу. 1979. Т. 19. С. 3-94. [2] А. А. Тарасов // УМН. 2002. Т. 57. № 5. С. 165-166. [3] Э. Б. Винберг // Изв. АН СССР. Сер. матем. 1990. Т. 54. № 1. С. 3-25. [4] А. Т. Фоменко // Матем. сб. 1981. T. 115. № 2. C. 263-280. [5] M. Nazarov, G. Olshanski // Comm. Math. Phys. 1996. V. 178. № 2. P. 433-506; arXiv: q-alg/9507003. [6] А. А. Тарасов // Матем. сб. 2000. T. 191. № 9. С. 115-122. [7] А. А. Тарасов // Матем. сб. 2003. Т. 194. № 7. С. 155-160. [8] Л. Г. Рыбников // Функц. анализ и его прил. 2003. Т. 37. № 2. С. 41-51.

Московский государственньй университет им. М. В. Ломоносова

E-mail: leo_rybnikov@mtu-net.ru
Представлено Э. Б. Винбергом Принято редколлегией 25.01.2005 